\title{
Simultaneous Optimization of Flight Control and Aerodynamic Shape for Aerocapture Vehicle
}

\author{
By Naohiko Honma and Kojiro SUZUKI \\ Department of Advanced Energy, Graduate School of Frontier Sciences, The University of Tokyo, Kashiwa, Japan
}

(Received July 17th, 2009)

\begin{abstract}
Aerocapture is expected to reduce the cost of the interplanetary exploration in the future because it utilizes aerodynamic drag to decelerate the vehicle rather than fuel-costly chemical propulsion. However, aerocapture mission involves lots of complicated difficulties such as quite narrow entry path angle, excess aerodynamic heating, aerodynamic load, and various uncertainties. It requires high levels of control technique and aerodynamic characteristics prediction. In this paper, the results of the simultaneous optimization of the flight control and aerodynamic shape for Mars aerocapture vehicle are presented. To maximize the payload mass, fuel mass for orbital transfer and TPS mass must be minimized. For this purpose, objective functions are set as follows: 1) minimize the delta- $\mathrm{V}$ for orbit insertion, 2) minimize the total heat load, 3) minimize both the delta- $\mathrm{V}$ and total heat load. Firstly, optimization was conducted only for the flight control, assuming the fixed aerodynamic shape and entry path angle. Secondary, simultaneous optimization was conducted. As a result, trade-off between delta-V and total heat load was clarified. Moreover, by executing simultaneous optimization, the guideline of designing aerocapture, which can minimize the objective function in each case 1)-3), was obtained.
\end{abstract}

Key Words: Aerocapture, Trajectory, Optimization, Genetic Algorithm

\section{Nomenclature}

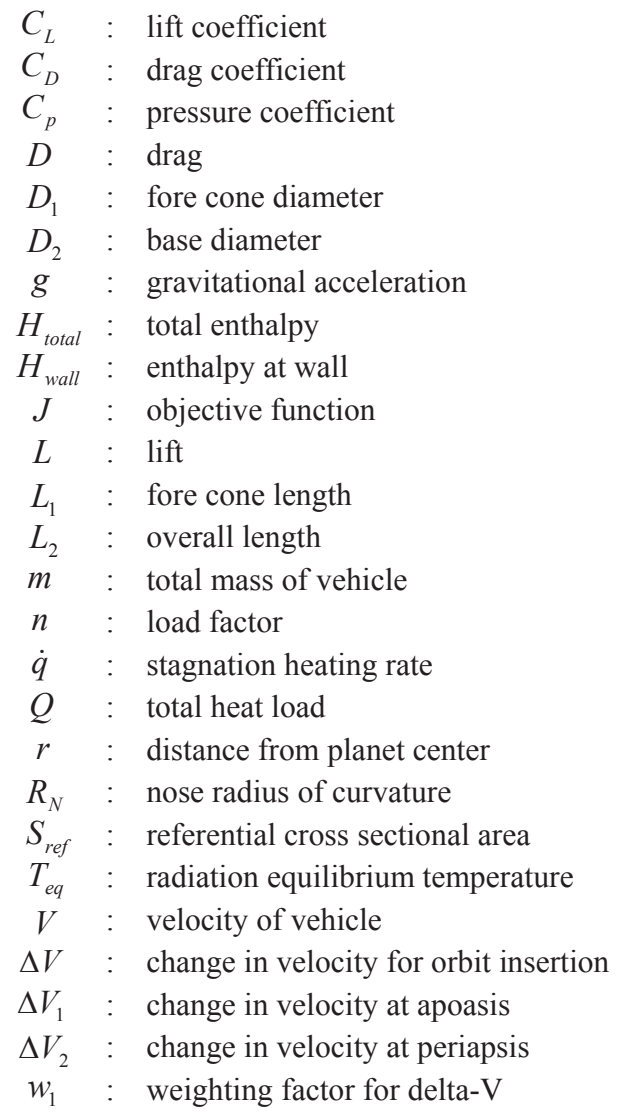

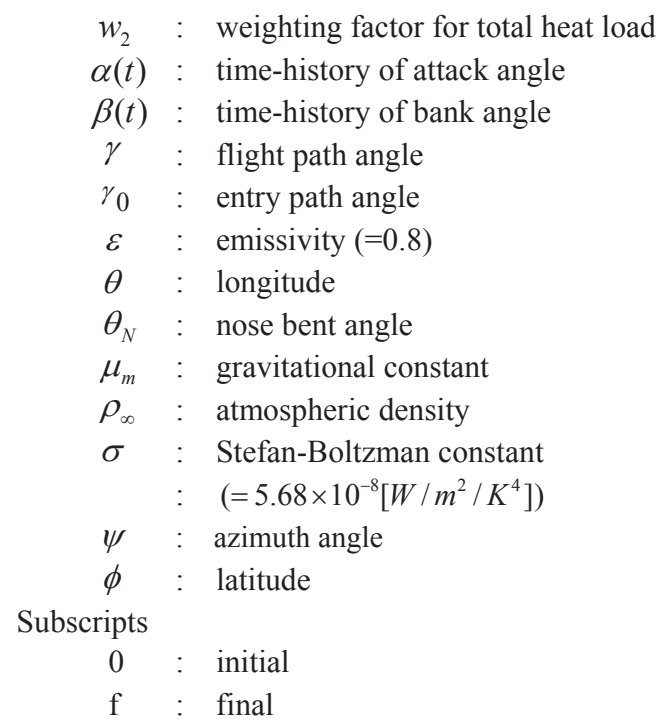

\section{Introduction}

Aerocapture technique ${ }^{1)}$ has the potential for providing substantial weight savings, because aerodynamic drag is used to remove enough kinetic energy instead of conventional fuel-costly chemical propulsion. It also enables us to complete orbital maneuvering in smaller time than aerobraking, which takes several weeks or several months to insert the vehicle into the target orbit. 
When an aerocapture mission is successfully conducted, a spacecraft is precisely inserted into a target orbit after a single atmospheric pass without any excess or insufficient deceleration. However, an aerocapture mission involves a lot of complicated technical difficulties. For example, the corridor width for the entry path angle is quite narrow and it strongly depends on the vehicle's aerodynamic characteristic and the entry velocity ${ }^{1,2)}$. During the atmospheric flight, the aerodynamic lift must be adequately controlled to obtain an optimized descending and ascending rate without losing necessary deceleration so that the vehicle can avoid the crash onto the ground even when the uncertainties in the atmospheric properties exist. Moreover, the aerodynamic heating that must be taken into account as one of the most critical problems depends on both the flight trajectory and the aerodynamic shape, that is, the atmospheric density, velocity, and the radius of a blunt nose. In addition to the uncertainties in the atmospheric density, we must consider the error in the entry angle, the model of the aerodynamic characteristics, the guidance and control. Such uncertainties and errors offer a significantly high risk on the aerocapture mission. Consequently, robustness is needed in both the vehicle shape design and the trajectory design to meet such uncertainties and errors ${ }^{3)}$

Therefore, in order to acquire enough deceleration while minimizing heat load, both the geometric parameters that determines aerodynamics and the time dependent control parameters that determines flight trajectory should be treated and be optimized simultaneously. For this reason, the objectives of this paper are:

1) To demonstrate the effectiveness of simultaneous optimization of aerodynamic shape and flight control for aerocapture vehicle. Trajectory-only optimization and simultaneous optimization are executed for three types of objective functions, that is, minimization of delta- $\mathrm{V}$ for the orbit insertion, minimization of the total heat load, and minimization of both delta- $\mathrm{V}$ and the total heat load.

2) To clarify the trade-off relation between the delta- $V$ minimization and the total heat load minimization for the trajectory-only optimization and the simultaneous optimization.

The optimization is conducted by using the computational code that has been originally developed for conceptual design of space planes using the Genetic algorithm (GA). Simultaneous optimization of the flight control and the aerodynamic shape is expected to give the solution for feasible aerocapture mission in the future.

\section{Mission}

Figure 1 shows an aerocapture flight profile schematic ${ }^{4)}$. The vehicle approaches the planet from a hyperbolic approach trajectory, shown at point 1 , and then enters the atmospheric interface, shown at point 2, within a predetermined range of the entry path angle. At point 3, bank angle modulation is initiated by the guidance. The drag on the vehicle provides the deceleration which is required to capture the vehicle into the desired orbit. The descent and ascent rates against the ground are controlled by the on-board guidance by rotating direction of the vehicle's lift vector about the velocity vector, so that the orbital elements at the atmospheric exit interface can match the ones required for insertion into the desired orbit. By point 5 , where the influence of aerodynamic forces is no longer significant, the kinetic energy is that required to capture the vehicle into the desired orbit. At point 7 and at point 8 , small delta- $\mathrm{V}$ burn is performed to raise the periapsis and to circularize the vehicle around the planet, respectively.

This paper considered following Mars-Aerocapture mission. The vehicle approaches the mars atmosphere at an entry speed of $5.7 \mathrm{~km} / \mathrm{s}$ at an altitude of $120 \mathrm{~km}$, which is calculated using patched conic approximation ${ }^{5}$, and the gross weight is $500 \mathrm{~kg}$. After single deep atmospheric pass, a small delta- $\mathrm{V}$ is performed and then the vehicle is inserted into a target science orbit at an altitude of $500 \mathrm{~km}$.

The problem is to find the optimum 1) entry path angle, 2) the control of the attack angle and bank angle during the atmospheric flight, and 3) the aerodynamic shape to achieve the minimum fuel consumption and minimum total heat load.

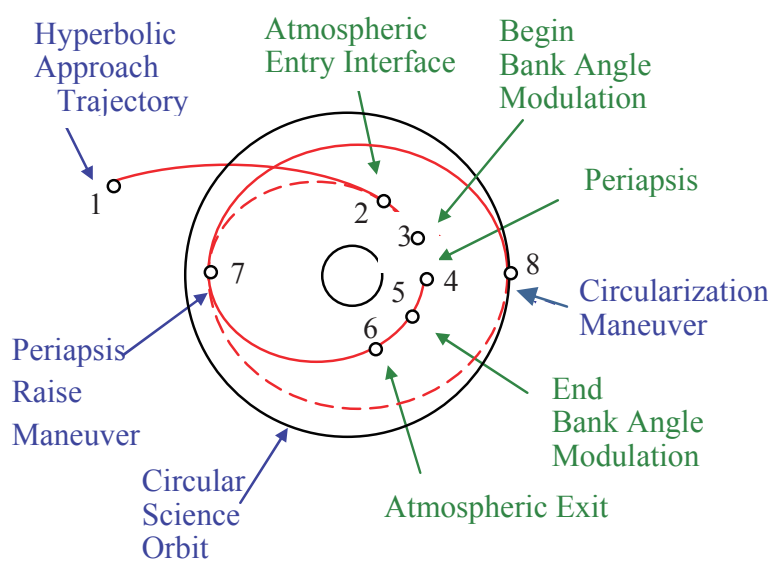

Fig. 1. Aerocapture flight schematic.

\section{Formulation for Optimization}

As already been mentioned, aerocapture requires high levels of control technique and aerodynamic characteristics because of its complicated mission requirement. The atmospheric entry trajectory strongly depends on the specifications of the vehicle and vice versa. Therefore it is expected that each aerodynamic shape has its own optimum flight trajectory and entry angle, so these parameters have to be treated as one set of design parameters. For constructing the conceptual design of aerocapture, flight control and aerodynamic shape need to be optimized simultaneously using an optimization method.

\subsection{Objective function}

Final goal of this work is to maximize the payload mass. Thus the fuel mass for orbital transfer and the thermal protection system mass must be minimized. In this paper, 
objective function for optimization problem is set as follows;

$$
\begin{gathered}
\min \quad J=w_{1} \times \Delta V+w_{2} \times Q \\
\Delta V=\left|\Delta V_{1}\right|+\left|\Delta V_{2}\right| \\
Q=\int_{t_{0}}^{t_{f}} \dot{q} d t
\end{gathered}
$$

In Eq. (1), delta- $V$, which is sum of the delta- $V$ at apoapsis and the delta- $\mathrm{V}$ at periapsis (Eq. (2)), is the propulsive requirements for post aerocapture. The delta- $\mathrm{V}$ at apoapsis is the change in velocity required for periapsis raise at point 7 . The delta- $\mathrm{V}$ at periapsis is for inserting into the target circular orbit at point $8 . \mathrm{Q}$ is the integrated heat load accumulated throughout atmospheric pass. The convective heating rate at stagnation point is obtained by Tauber's relation ${ }^{6)}$ (Eq. (4)).

$$
\dot{q}=1.35 \times 10^{-10} \sqrt{\frac{\rho_{\infty}}{R_{N}}} V^{3.01} \times\left(1-\frac{H_{\text {wall }}}{H_{\text {total }}}\right)
$$

\subsection{Control variables}

Control variables of trajectory are;

- initial flight path angle: $\quad \gamma_{\min } \leq \gamma_{0} \leq \gamma_{\max }$

- time-history of angle of attack: $\quad \alpha_{\min } \leq \alpha(t) \leq \alpha_{\max }$

- time-history of bank angle: $\quad \beta_{\min } \leq \beta(t) \leq \beta_{\max }$

It is assumed that some control surfaces that attached to the body enable the vehicle to control its attitude.

\subsection{Design variables}

The shape considered is the spherically blunted biconic shown in Fig.2, which is defined by 6 parameters:

$$
\begin{array}{ll}
\text { - fore cone length: } & L_{1 \min } \leq L_{1} \leq L_{1 \max } \\
\text { - overall length: } & L_{2 \min } \leq L_{2} \leq L_{2 \max } \\
\text { - fore cone diameter: } & D_{1 \text { min }} \leq D_{1} \leq D_{1 \max } \\
\text { - base diameter: } & D_{2 \min } \leq D_{2} \leq D_{2 \max } \\
\text { - nose radius: } & R_{N \text { min }} \leq R_{N} \leq R_{N \max } \\
\text { - nose bent angle: } & \theta_{N \text { min }} \leq \theta_{N} \leq \theta_{N \max }
\end{array}
$$

Upper limits of design variables are constrained by the size of H-IIB Rocket fairing ${ }^{6}$.

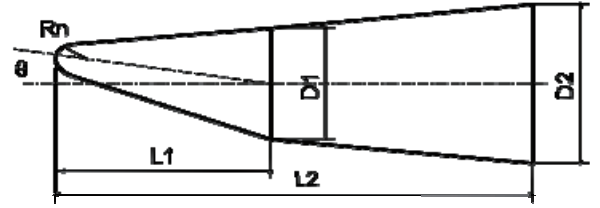

Fig. 2. Design variables.

\subsection{Constraints}

The radiation equilibrium temperature at the stagnation point and the load factor are considered as constraints. The former is calculated by Eq. (7). Acceptable values for control variables, design variables, and constraint conditions are listed in Table 1.

$$
\begin{aligned}
T_{e q} & \leq T_{e q \max } \\
n & \leq n_{\max } \\
T_{e q} & =\left(\frac{\dot{q}}{\varepsilon \sigma}\right)^{0.25}
\end{aligned}
$$

Table 1. Acceptable values.

\begin{tabular}{|l|c|c|}
\hline & minimum & maximum \\
\hline$\gamma_{0}[\mathrm{deg}]$ & -30 & 0 \\
\hline$\alpha(t)[\mathrm{deg}]$ & 0 & 40 \\
\hline$\beta(t)[\mathrm{deg}]$ & -90 & 90 \\
\hline$L 1[\mathrm{~mm}]$ & 0 & 4000 \\
\hline$L 2[\mathrm{~mm}]$ & 0 & 4000 \\
\hline$D 1[\mathrm{~mm}]$ & 0 & 4000 \\
\hline$D 2[\mathrm{~mm}]$ & 0 & 4000 \\
\hline$R_{N}[\mathrm{~mm}]$ & 0 & 2000 \\
\hline$\theta[\mathrm{deg}]$ & 0 & 10 \\
\hline$T e q[\mathrm{~K}]$ & - & 1500 \\
\hline$n[\mathrm{G}]$ & - & 8 \\
\hline
\end{tabular}

\section{Mathematical Models for Analysis}

\subsection{Vehicle flight dynamics}

For describing the dynamics of the vehicle, the three-dimensional, non-sideslip and point mass model for an un-powered flight over a non-rotating spherical Earth is used.

$$
\begin{gathered}
\dot{r}=V \sin \gamma \\
\dot{\theta}=V \cos \gamma \cos \psi / r \cos \phi \\
\dot{\phi}=V \cos \gamma \sin \psi / r \\
\dot{V}=-D / m-g \sin \gamma \\
\dot{\gamma}=(L / m V) \cos \beta-(g / V-V / r) \cos \gamma \\
\dot{\psi}=(L / m V) \cdot \sin \beta / \cos \gamma-V \cos \gamma \tan \phi \cos \psi / r
\end{gathered}
$$
where,

$$
\begin{gathered}
g=\mu_{m} / r^{2} \\
L=\frac{1}{2} \rho V^{2} C_{L} S_{r e f} \\
D=\frac{1}{2} \rho V^{2} C_{D} S_{r e f}
\end{gathered}
$$

\subsection{Aerodynamics}

Modified Newtonian theory is used in this work to approximate the pressure at each point on the body surface. According to this theory, the pressure coefficient $\mathrm{Cp}$ is a function of the angle between the velocity vector and the unit vector normal to the panel of the body ${ }^{8)}$.

$$
C_{p}=C_{P 0}\left(\frac{\vec{V}}{|\vec{V}|} \cdot \frac{d \vec{s}}{|d \vec{s}|}\right)^{2}
$$

\section{Optimization Method by GA}

Genetic algorithm (GA) is search algorithm based on the mechanism of natural selection and genetic mechanism. They are not sensitive to initial guess, and good performance to large-scale nonlinear complex global optimization problems. They don't require derivative or continuity of a function to be evaluated, but only use objective function information. Moreover, they are simple, fast converging and easy to implement and simulate. For these reasons, GA is applied to this paper. As a crossover and generation alternation method, UNDX (Unimodal Normal Distribution Crossover) ${ }^{9)}$ and MGG(Minimal Generation Gap) ${ }^{10)}$ are used. GA parameters are shown in Table 2. 
Table 2. GA parameters.

\begin{tabular}{|c|c|}
\hline Population size & 30 \\
\hline Generation & 500 \\
\hline Crossover & 5 \\
\hline
\end{tabular}

\section{Results}

Firstly, optimization is conducted only for the flight control, assuming the fixed aerodynamic shape and fixed entry path angle $\left(\gamma_{0}=-11.5 \mathrm{deg}\right)$.

Secondly, simultaneous optimization of flight control and aerodynamic shape is conducted for variable entry path angle. To analyze the tendency of the solution, optimizations are conducted for each objective functions as shown below (case $1 \sim$ case 3 ). Case 1 corresponds to the minimization of the fuel mass required for orbit insertion irrespectively of the Thermal Protection System (TPS) mass. In case 2, only the TPS mass is considered for optimization. In case 3, both the fuel mass and TPS mass are considered with weighting factors $\mathrm{w}_{1}$ and $\mathrm{w}_{2}\left(\mathrm{w}_{1}: \mathrm{w}_{2}=1: 1\right)$.

$$
\begin{array}{lll}
\text { case 1) } & \min & J=\Delta V \\
\text { case 2) } & \min & J=Q \\
\text { case 3) } & \min & J=w_{1} \times \Delta V+w_{2} \times Q
\end{array}
$$

\subsection{Trajectory optimization}

Trajectory optimization was conducted for assumed shape which has aerodynamic coefficient shown in Fig.3. Results for each objective function are listed in Table 3 . It contains the data of delta- $\mathrm{V}$, total heat load, load factor, radiation equilibrium temperature, and the amount of time to exit atmosphere. The time history of altitude and time history of radiation equilibrium temperature are shown in Fig.4, and Fig.5, respectively. Figure 6 shows the relation between fuel mass fraction and TPS mass fraction calculated by following equation $^{11)}$. In eq (18), it is assumed that $I_{\mathrm{sp}}$ is $300 \mathrm{sec}$.

$$
\begin{gathered}
F U E L \%=\frac{M_{\text {fuel }}}{M_{\text {spacecraft }}}=1.0-\exp \left(-\frac{\Delta V}{g_{0} \cdot I_{s p}}\right) \\
T P S \%=\frac{M_{T P S}}{M_{\text {spacecraft }}}=0.091 \times Q^{0.51575}
\end{gathered}
$$

For comparison, the case without optimization is also considered ("case 0" in Table 1). In this case, it is assumed that the vehicle flies at constant attack angle and bank angle.

It can be seen that, in Fig.4, the vehicle controls the altitude to minimize its objective function. For case 1 , in order to obtain sufficient deceleration, the vehicle takes a longer time to fly high-density region than case 2 and case 3 . However, this flight increases the total heat load. On the other hand, in case 2, the vehicle tends to be captured in the shortest possible time in order to avoid total heat load. In case 3 , the delta- $V$ is successfully decreased with some amount of heat load reduction. It can be seen that the assumed aerodynamic shape has the trade-off between fuel mass and TPS mass as shown in Fig.6. From the viewpoint of mass fraction, the fuel mass fraction of the assumed aerodynamic shape has stronger sensitivity to each objective function rather than TPS mass fraction. And also it can be seen that, in Fig.4, flight trajectories are almost identical in the descent phase. It shows that descent phase and its minimum altitude strongly depend on the entry angle for the assumed condition.

Table 3 also shows that all the cases are violating the constraints of radiation equilibrium temperature and load factor. Throughout the whole generation of GA, no solutions satisfied the setup constraints. Particularly, in Fig.5, radiation equilibrium temperatures for all the cases are almost the same value $(1760 \sim 1785 \mathrm{~K})$. It appears that heating rate is quite sensitive to the nose radius and entry angle rather than flight control.
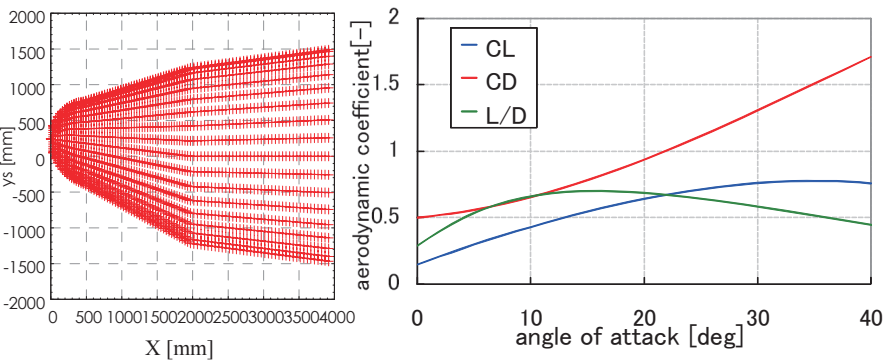

Fig. 3. Assumed shape $\left(\mathrm{R}_{\mathrm{N}}=600 \mathrm{~mm}\right)$ and aerodynamic coefficient.

Table 3. Results for each objective function.

\begin{tabular}{|c|c|c|c|c|}
\hline & case 0 & case1 & case2 & case3 \\
\hline$\Delta V[m / s]$ & 609 & 141 & 357 & 146 \\
\hline$Q\left[M J / m^{2}\right]$ & 33.5 & 42.1 & 31.6 & 35.4 \\
\hline$n[G]$ & 9.56 & 10.4 & 11.3 & 11.7 \\
\hline $\mathrm{T}_{\mathrm{eq}}[\mathrm{K}]$ & 1784 & 1778 & 1759 & 1762 \\
\hline Time $[\mathrm{sec}]$ & 282 & 483 & 309 & 441 \\
\hline
\end{tabular}

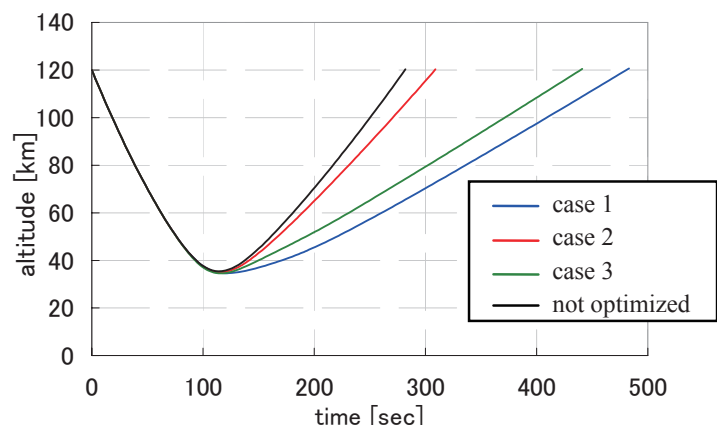

Fig. 4. Time-history of altitude for each objective function.

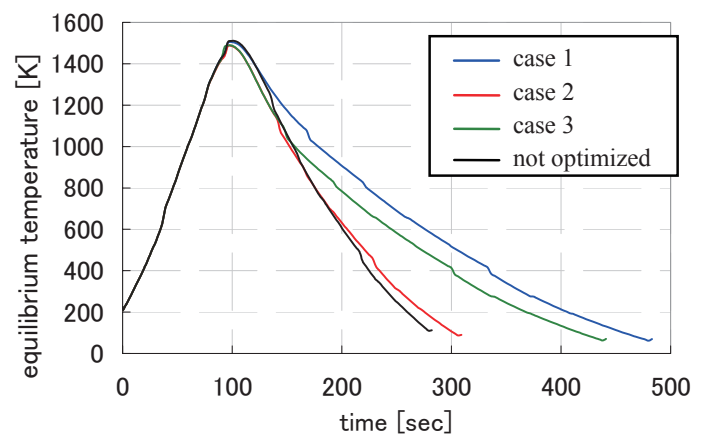

Fig. 5. Time-history of equilibrium temperature for each objective function. 


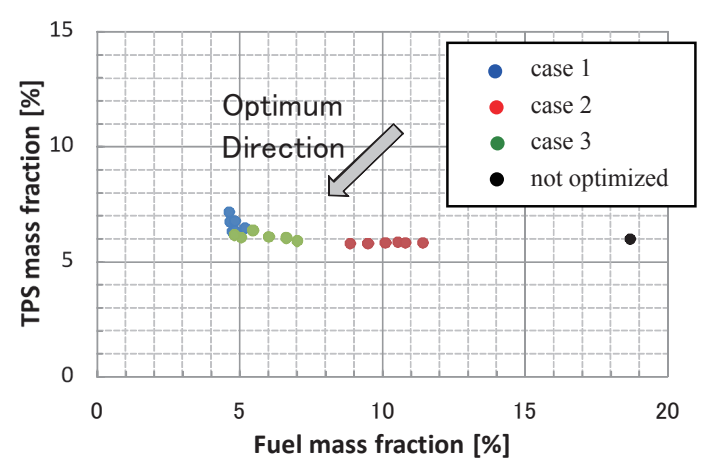

Fig. 6. Fuel mass fraction vs. TPS mass fraction.

\subsection{Simultaneous optimization}

The results of simultaneous optimization for each objective function are listed in Table 4. Fuel mass fraction, TPS mass fraction, the sum of payload and structural mass fraction for each solution are listed in Table 5. In Table 5, "chemical" means the conventional all-chemical propulsion deceleration assuming that $I_{\text {sp }}$ is $315 \mathrm{sec}$. The time history of altitude and time history of equilibrium temperature are shown in Fig.7 and Fig. 8, respectively. The optimized shapes and their aerodynamic coefficient for each objective function are shown in Figs 9, 10, and 11.

Table 4 shows that each objective function was minimized much lower than the case of optimizing the flight control only. It also appears that no one violates the constraints. For case 1, vehicle produces and controls a small negative lift by the inverted flight. It makes the vehicle stay at high-density region and acquire lower delta- $\mathrm{V}$. However, smaller nose radius $(870$ $\mathrm{mm}]$ ) and longer duration of atmospheric flight time increase the total heat load. Moreover, this shape will be insufficient volumetric efficiency, which negatively affects the reduction of fuel mass. Volumetric efficiency is not included as a constraint in this analysis. Neglecting it allows the solutions to have a large wet surface area and a small volume. However, in this analysis, solutions came close to a sphere shape because optimized shape tends to have a lower L/D and a larger nose radius. For case 2, it maximizes the fore cone diameter, base diameter, and nose radius to avoid the aerodynamic heating and to decelerate at higher altitude. And also, delta-Vis increased because of entering the atmosphere with a shallow initial flight path angle. For case 3, Fig.11 is the Pareto solution. The vehicle enters the atmosphere deeper than the others as can be seen in Fig.7. As soon as it gets enough deceleration, it goes upward rapidly by producing and controlling the lift vector to avoid staying at higher density region. In this case, the optimized aerodynamic shape that has maximum $\mathrm{L} / \mathrm{D}$ of 0.2 , maximum $\mathrm{C}_{\mathrm{D}}$ of 1.0 , and the ballistic coefficient of $39.8 \mathrm{~kg} / \mathrm{m}^{2}$ was obtained. However, L/D of 0.2 may give a small aerocapture corridor due to uncertainties in atmospheric density. Therefore, from a viewpoint of corridor width, some constraints on L/D should have been included in the optimization.

Table 5 shows that the solution of case 3 maximized the payload and structural mass fraction, which is $90.9 \%$. This percentage of case 3 is nearly twice as much as that of conventional all-chemical propulsion.

Table 4. Results for each objective function.
\begin{tabular}{|c|c|c|c|}
\hline & case 1 & case 2 & case 3 \\
\hline$\gamma 0[\mathrm{deg}]$ & -9.31 & -8.83 & -9.97 \\
\hline$\Delta V[\mathrm{~m} / \mathrm{s}]$ & 112 & 1372 & 131 \\
\hline$Q\left[\mathrm{MJ} / \mathrm{m}^{2}\right]$ & 39.5 & 12.61 & 21.13 \\
\hline$n[\mathrm{G}]$ & 3.64 & 2.19 & 6.56 \\
\hline $\mathrm{T}_{\mathrm{eq}}[\mathrm{K}]$ & 1198 & 919 & 1141 \\
\hline Time $[\mathrm{sec}]$ & 735 & 333 & 513 \\
\hline
\end{tabular}

Table 5. Mass fraction.

\begin{tabular}{|l|c|c|c|c|}
\hline & case 1 & case 2 & case 3 & chemical \\
\hline FUEL [\%] & 3.7 & 37.3 & 4.4 & 46.7 \\
\hline TPS [\%] & 6.5 & 3.6 & 4.7 & - \\
\hline $\begin{array}{l}\text { Payload \& } \\
\text { Structure[\%] }\end{array}$ & 89.8 & 59.1 & 90.9 & 53.3 \\
\hline
\end{tabular}

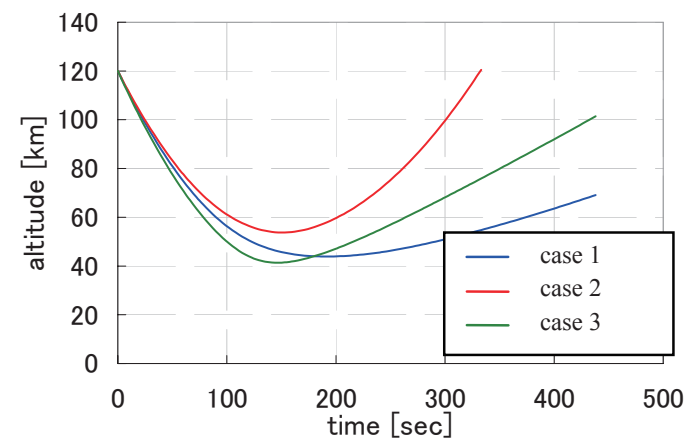

Fig. 7. Time-history of altitude for each objective function.

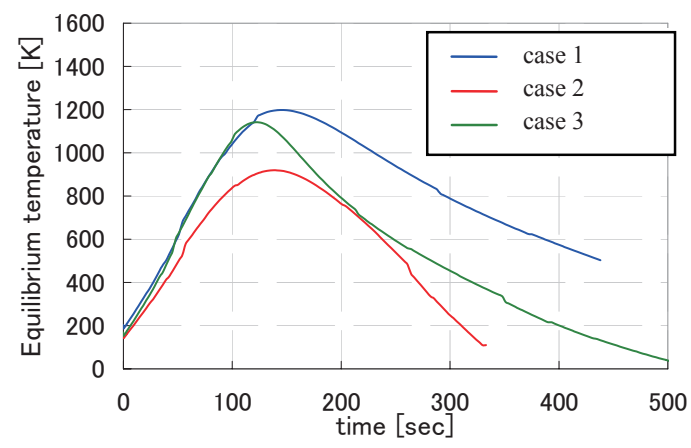

Fig. 8. Time-history of equilibrium temperature for each objective function.

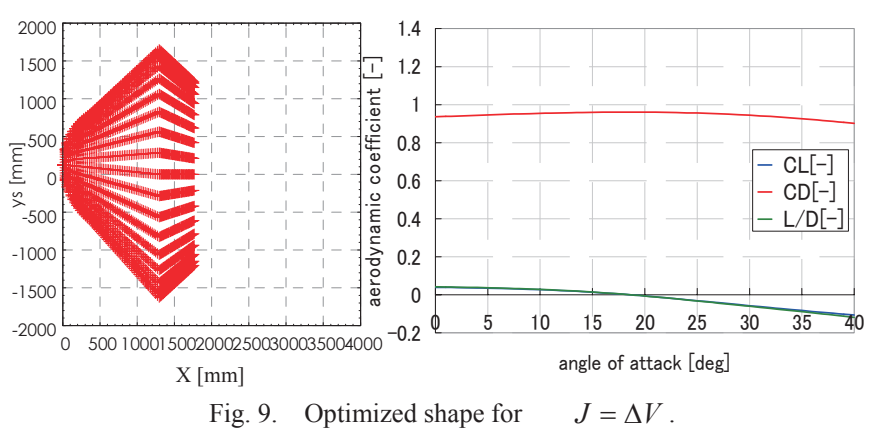



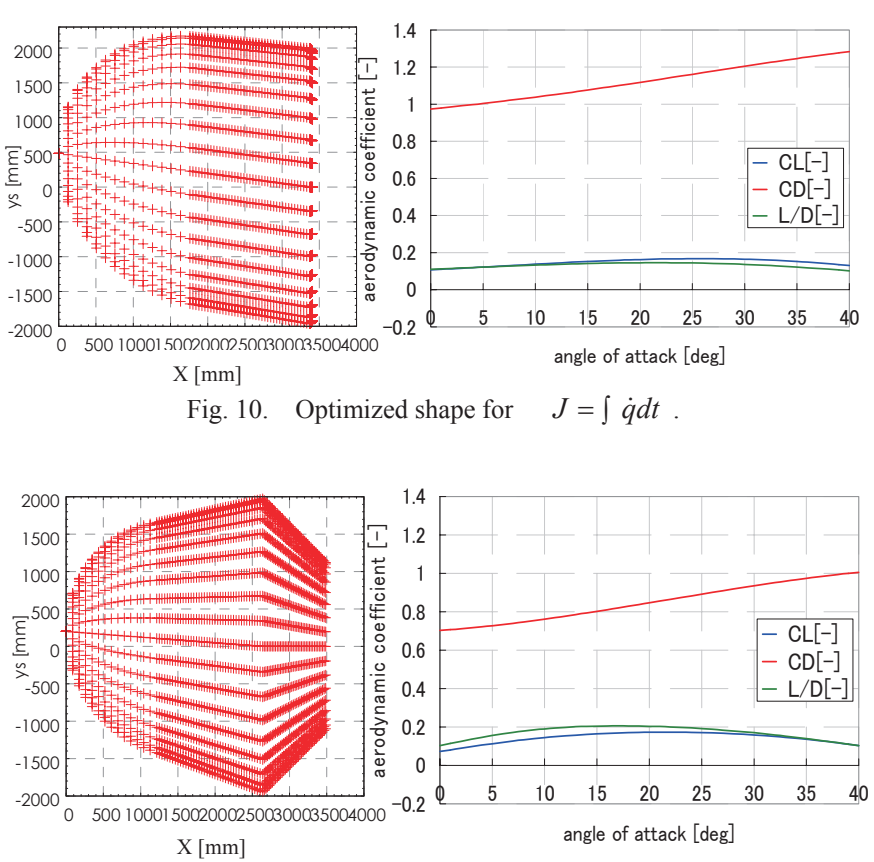

Fig. 11. Optimized shape for $J=w_{1} \times \Delta V+w_{2} \times \int \dot{q} d t$.

\section{Conclusions}

Simultaneous optimization of flight control and aerodynamic shape was conducted. Objective functions are set as follows: 1) minimize a delta- $\mathrm{V}$ for orbit insertion, 2) minimize a total heat loads 3) minimize both delta- $\mathrm{V}$ and total heat loads. Compared with a trajectory-only optimization, the results of simultaneous optimization indicated that it could decrease the objective function values for all the cases. Therefore, the effectiveness of this method is demonstrated.

The results of flight control optimization for fixed shape and fixed entry angle indicate the existence of trade-off between fuel mass for delta- $V$ and TPS mass for total heat load. The shape and the flight path angle which were arbitrary given as the initial conditions were found to violate the constraints with respect to the radiation equilibrium temperature and the load factor. By introducing optimization to both parameters, these constraints are completely satisfied, and the required fuel mass and the TPS mass are minimized as well. Consequently, the results of simultaneous optimization show that this method can minimize the required fuel mass with some reduction in the TPS mass. In this sense, single objective solutions such as the case 1 (only fuel mass) and the case 2 (only TPS mass) are not appropriate.

\section{Appendix Accuracy of Newtonian Theory}

Navier-Stokes equation is solved for the obtained shape of case 3 assuming the 2 dimensional and axisymmetric body. The computational domain is shown in Fig.12. Two-step Runge-Kutta method and Yee's symmetric TVD ${ }^{12)}$ scheme are used as a time integration and as a discretization of convective term, respectively. Figure 13 shows the pressure contour. The drag coefficient is 0.646 , whereas drag coefficient which is calculated by modified Newtonian theory is 0.703 . Consequently, the estimation error by the Newtonian theory is expected to be less than $10 \%$. The error in estimation of the aerodynamic characteristics should be considered in the case of optimization with robustness for various uncertainties in the analysis models.

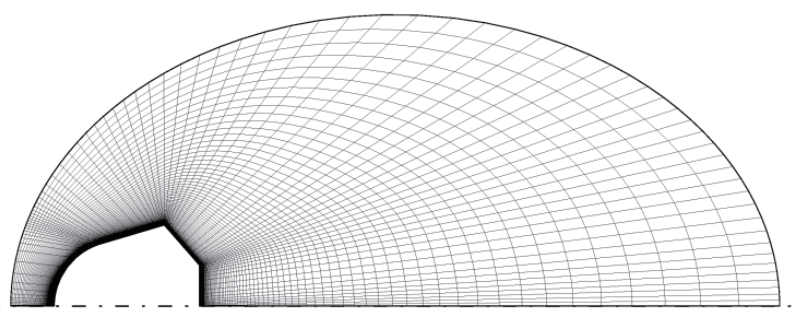

Fig. 12. Computational grid $(81 \times 101)$.

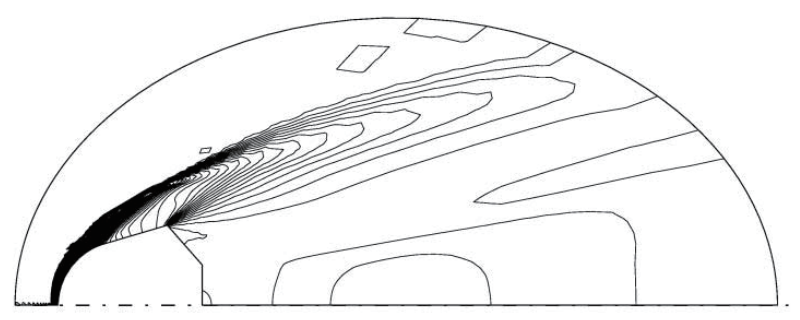

Fig. 13. Pressure contour.

\section{References}

1) Walberg, G. D.: A Survey of Aeroassisted Orbit Transfer, $J$. Spacecraft and Rockets, 22 (1985), pp.8-9.

2) Craig, S.: A Parametric Study of Aerocapture for Mission to Venus, AIAA paper, 2002-4500.

3) Way, D. W., et al.: Aerocapture Simulation and Performance for the Titan Explorer Mission, AIAA paper, 2003-4951.

4) Lockwood, M. K.: Titan Aerocapture System Analysis, AIAA paper, 2003-4799.

5) Shibahara, M., Kida, T.: Introduction to the Space Engineering (2) (in Japanese) , Baifukan, Tokyo, (1998), pp.117-128

6) Tauber, M. E., Bowles, J. V. and Yang, L.: Use of Atmospheric Braking during Mars Missions, J.Spacecraft and Rockets, 27 (1990), pp.514-521.

7) http://www.jaxa.jp/projects/rockets/h2b/index j.html

8) Anderson, J. D., Jr.: Hypersonic and High Temperature Gas Dynamics, McGraw-Hill Book Company, New York, (1989), pp.45-56.

9) Ono, I., Satoh, H., Kobayashi, S.: A Real-Coded Genetic Algorithm for Function Optimization using the Unimodal Normal Distribution Crossover, J. Japanese Society for Artificial Intelligence, 14 No.6, (1999), pp. 1146-1155.

10) Satoh, H., Ono, I., Kobayashi, S.: A New Generation Alternation Model of Genetic Algorithms and Its Assessment, J.Japanese Society for Artificial Intelligence, 12 No.5, (1997), pp. 734-744.

11) Glen, J. Brown., Erin, Richardson.: Minimum-Mass Design for Titan Aerocapture, AIAA paper, 2005-1637.

12) Yee, H. C.:A Class of High-Resolution Explicit and Implicit Shock-Capturing Methods, NASA TM 101088, 1989. 